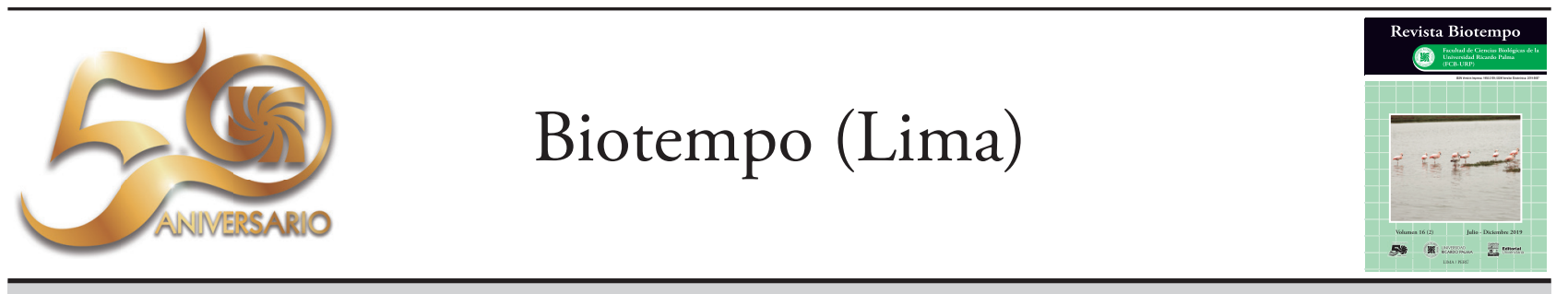

ORIGINAL ARTICLE / ARTÍCULO ORIGINAL

PROSPECTIVE PLANNING FOR TRAINING DEVELOPMENT OF LEARNING ON METHODOLOGY OF SCIENTIFIC RESEARCH IN UNIVERSITY STUDENTS THROUGH ACCELERATED MODALITY

\title{
PLANIFICACIÓN PROSPECTIVA PARA EL DESARROLLO FORMATIVO DEL APRENDIZAJE SOBRE METODOLOGÍA DE LA INVESTIGACIÓN CIENTÍFICA EN ESTUDIANTES UNIVERSITARIOS MEDIANTE MODALIDAD ACELERADA
}

George Argota-Pérez ${ }^{1}$; Ramiro M. Yallico-Calmett ${ }^{2 a}$; Kony L. Duran LLaro ${ }^{3}$; José Iannacone ${ }^{4,5}$; Rosa A. Aquije-García ${ }^{2 a} \&$ Flor A. Lavanda-Reyes ${ }^{2 b}$

1 Centro de Investigaciones Avanzadas y Formación Superior en Educación, Salud y Medio Ambiente "AMTAWI", Puno-Perú. george.argota@gmail.com

2 Universidad Nacional San Luis Gonzaga (UNICA). Ica-Perú. a Facultad de Ciencia de la Educación y Humanidades. ereyece@hotmail.com; raquije13@hotmail.com b Facultad de Administración. angielavanda@gmail.com

3 Escuela de Posgrado. Universidad César Vallejo (UCV). Trujillo-Perú. kony27_teacher@hotmail.com

4 Laboratorio de Parasitología. Facultad de Ciencias Biológicas. Universidad Ricardo Palma (URP). Lima-Perú. joseiannacone@gmail.com

5 Laboratorio de Ecología y Biodiversidad Animal. Facultad de Ciencias Naturales y Matemática. Universidad Nacional Federico Villarreal (UNFV). Lima-Perú;

Author for correspondence: george.argota@gmail.com

\section{ABSTRACT}

The aim of study was to evaluate the prospective planning for the formative development of learning about the methodology of scientific research in university students through accelerated modality. A differential prospective route was considered through accelerated training between April 22 and 26 (replica I) and from April 29 to May 3, 2019 (replica II) which was directed to, students of the 10th academic cycle belonging to the Faculty of Civil Engineering of National University San Luis Gonzaga of Ica, Peru. Two variables of interest were considered for analysis: 1st), responsibility before the social recognition of graduation (RSRG) and 2nd), number of project proposals to present (NPPP). The RSRG was evaluated by means of assistance and the advance on the practical activities developed in class. The RSRG was evaluated through assistance and progress on practical activities developed in class. The NPPP was evaluated by the percentage of compliance on the methodological structures formulated according to the format of research thesis project. The attendance and advance on the practical activities developed in class by the students decreased by day in replica I but remained in replica II. The NPPP in replica I was low (3) compared to replica II (18). It was considered that the efficient 
prospective route for learning the methodology of the research was the production and research on the theme proposal to be developed according to the recognition of the variables to be measured by the students.

Keywords: learning - university students - research training - research methodology - prospective planning

\section{RESUMEN}

El objetivo del estudio fue evaluar la planificación prospectiva para el desarrollo formativo del aprendizaje sobre la metodología de la investigación científica en estudiantes universitarios mediante modalidad acelerada. Se consideró una ruta prospectiva diferencial mediante la capacitación con modalidad acelerada entre los días 22 y 26 de abril (réplica I) y del 29 de abril al 3 de mayo de 2019 (réplica II) la cual fue dirigida a, estudiantes del 10mo ciclo académico perteneciente a la Facultad de Ingeniería Civil de la Universidad Nacional San Luis Gonzaga de Ica, Perú. Dos variables de interés fueron consideradas para su análisis: 1ro), responsabilidad ante el reconocimiento social de graduación (RRSG) y 2do), número de propuestas de proyectos a presentar (NPPP). La RRSG se evaluó mediante la asistencia y el avance sobre las actividades prácticas desarrolladas en clase mientras que, NPPP se evaluó por el porcentaje de cumplimiento sobre las estructuras metodológicas formuladas según el formato del proyecto de tesis de investigación. La asistencia y el avance sobre las actividades prácticas desarrolladas en clase por los estudiantes disminuyeron por día en la réplica I pero se mantuvieron en la réplica II. El NPPP en la réplica I fue bajo (3) en comparación con la réplica II (18). Se consideró que la ruta prospectiva eficiente para el aprendizaje de la metodología de la investigación fue la producción e investigación sobre la propuesta de tema a desarrollar según el reconocimiento de las variables a medir por los estudiantes.

Palabras clave: aprendizaje - estudiantes universitarios - formación investigativa - metodología de la investigación planificación prospectiva

\section{INTRODUCCIÓN}

Uno de los procesos más complejo de la cotidianidad, es la toma de decisiones con base a la planificación (Luo, 2018). La planificación resulta esencial para alcanzar procedimientos eficientes (Wang \& Yang, 2014; Travis et al., 2014) y con frecuencia, pensar en el logro de resultados deseados donde podrían incluirse multitudes de situaciones y rutas de orientación al menos, desde la evaluación sobre dos criterios: $1^{\mathrm{ro}}$ ), valor objetivo de las acciones a realizarse y $2^{\mathrm{do}}$ ), mostrar capacidades para conseguirse (Fisher, 2018).

Cualquier escenario puede entenderse como un filtro cognitivo basado en historias de procesos para ofrecer y recibir algún tipo de lógica (MacKay \& McKiernan, 2016), siendo la actividad docente e investigación universitaria un ejemplo para el progreso de la planificación estratégica de escenarios sociales (Abrahamson, 1991; Gill \& Whittle, 1993) donde cualquier análisis diferencial de la planificación se basa en la gestión no solo estratégica sino, práctica (Cummings \& Daellenbach, 2009; sin embargo, solo el reconocimiento sobre la gestión estratégica de la planificación dependerá de, cuánto exista en innovación (Bradfield et al., 2016).

Según la Comisión Económica para América Latina y el Caribe, la planificación surge a mediados del siglo XX donde la crisis que se presentó desde la década de 1970 y posterior deudas existentes, debilitaron la articulación entre el plan, presupuesto y resultados, es decir, la distinción entre el sujeto (Estado) de la planificación y el objeto (realidad económica-social) de la intervención deja de coexistir (reconocida como planificación normativa) (Mattar \& Cuervo, 2017).

Para Mattus (1972), lo transcendental estuvo en explicar las situaciones presentadas independientemente del diagnóstico. Otra concepción de la planificación, es la estratégica la cual proviene de la administración del desarrollo y escuela de negocios donde sus preceptos se basan en planear, organizar, dirigir, coordinar y controlar (Jiménez, 1982).

Considerándose lo anterior, lo más relevante de la planificación es la planificación prospectiva. Entre 1955 
y 1960, el pensador o pionero en acuñar el término fue, Gastón Berger, considerado el padre de la prospectiva (Mera, 2014) quien señaló que, la prospectiva es una actitud visionaria y que consta de cinco características: $1^{\mathrm{ro}}$ ) visión de fututo con alta calidad, $2^{\mathrm{do}}$ ) visión a largo plazo, $3^{\text {ro }}$ ) visión sistémica, $4^{\text {to }}$ ) visión de profundidad basada en fundamentos metodológicos sustentables donde se permita aumentar el conocimiento mediante la toma de decisiones y $5^{\text {to }}$ ) visión diferente, es decir, aceptar lo que no se había pensado asumiendo los riesgos.

Para Jouvenel (1964), la prospectiva es anticiparse o explorar futuros posibles, probables o deseables. En el caso de Pierre Massé en1962, representante de la propia escuela francesa, define el enfoque prospectivo adoptado donde se trata de extraer del ámbito de lo posible, algunas figuras del fututo que sean inteligibles para el espíritu y prácticas para la acción.

Los mayores exponentes de la prospectiva desde el punto de vista estratégico son Goder \& Durance (2011), quienes describen métodos formales y cuantitativos, aplicando herramientas informáticas, además, de la previsión humana y social.

A partir, de disimiles informaciones disponibles en la literatura científica que muestran diferentes esquemas para abordar la visión y construcción de un futuro mejor (Ingvar, 1985; Martin, 1995; Mojica, 2006; Anderson, 2012; McLelland et al., 2015; Patel, 2016; es que resulta imprescindible, la preparación temprana en la formación metodológica de estudiantes universitarios donde las evaluaciones prácticas deben ser continuas, pues como seńala Lisle (2015) la medición sistemática garantiza la preparación de los estudiantes, además, constituye el único recurso de validez para el logro de su formación (Carless, 2011; Yin \& Buck, 2015) y probablemente, pueda servir como inicio a la visión del futuro desde su profesión. Hoffmann \& Koifman (2013) indican la necesidad de buscar modelos que sean capaces de generar profesionales reflexivos y competentes y parece no existir dudas que, la prácticas y entrenamiento de evaluación permiten otorgan dominio, oportunidades y aceptables resultados para el aprendizaje (Hansen \& Ringdal, 2018).

El objetivo del estudio fue evaluar la planificación prospectiva para el desarrollo formativo del aprendizaje sobre la metodología de la investigación científica en estudiantes universitarios mediante modalidad acelerada.

\section{MATERIALES Y MÉTODOS}

Considerándose, no ser habitual en el Perú, la orientación y acompańamiento docente para lograr en los estudiantes universitarios la sustentación de tema de investigación seleccionado bajo la transformación de los problemas sociales relacionados con la especialidad y que, pudieran estar debidamente reconocibles en la líneas de investigación se genera, cómo principal dificultad, la incertidumbre durante el posible tiempo a presentar el proyecto de tesis de investigación. Entre el reconocimiento de las posibles rutas para presentar el proyecto de tesis de investigación que los autores consideran se encuentran las siguientes (Tabla 1):

Tabla 1. Rutas para presentar el proyecto tesis de investigación / estudiantes universitarios.

\begin{tabular}{|c|c|}
\hline Positiva & Negativa \\
\hline $\begin{array}{l}\text { Responder (aunque en tiempo demorado según el análisis } \\
\text { observado) la estructura metodológica del formato de tesis } \\
\text { de investigación mediante asesorías y consultorías internas } \\
\text { como externas }\end{array}$ & $\begin{array}{l}\text { Pagar por el servicio de redacción sobre el formato del } \\
\text { proyecto de tesis de investigación }\end{array}$ \\
\hline \multirow{3}{*}{$\begin{array}{l}\text { Responder, luego de capacitaciones en condiciones de pos- } \\
\text { egreso universitario la estructura metodológica del formato } \\
\text { de tesis de investigación }\end{array}$} & $\begin{array}{l}\text { Pagar por el servicio de redacción sobre la tesis de } \\
\text { investigación }\end{array}$ \\
\hline & $\begin{array}{l}\text { Sobornar a los miembros jurados para la aprobación } \\
\text { sobre propuestas de investigación a presentarse }\end{array}$ \\
\hline & $\begin{array}{l}\text { Presentar facilidades como exámenes de evaluativos para } \\
\text { la aprobación durante un periodo determinado }\end{array}$ \\
\hline
\end{tabular}

Se calificó en este estudio como ruta prospectiva diferencial, una capacitación con modalidad acelerada entre los días 22 y 26 de abril de 2019 y dirigida a, estudiantes del $10^{\mathrm{mo}}$ ciclo académico perteneciente a la Facultad de Ingeniería Civil de la Universidad Nacional San Luis Gonzaga de Ica, Perú. Se consideró dos variables de interés para su análisis: $1^{\mathrm{r}}$ ), responsabilidad ante el reconocimiento social de graduación (RRSG) y $2^{\text {do }}$ ), número de propuestas de proyectos a presentar (NPPP). La RRSG se evaluó mediante la asistencia y el avance sobre las actividades prácticas desarrolladas en clase mientras que, NPPP se evaluó por el porcentaje de cumplimiento 
sobre las estructuras metodológicas formuladas según el formato del proyecto de tesis de investigación (viernes: último día de curso) vigente en la Facultad de Ingeniería Civil.

Se planteó como hipótesis en el estudio lo siguiente:

- Ho: el número de propuesta de proyectos a presentar (NPPP) depende de la responsabilidad ante el reconocimiento social de graduación (RRSG) por los estudiantes.

- Hi: el número de propuesta de proyectos a presentar (NPPP) no depende de la responsabilidad ante el reconocimiento social de graduación (RRSG) por los estudiantes.

Se utilizó el programa estadístico profesional Statgraphics Centurion XVIII donde el estadístico descriptivo de tendencia relativa fue la frecuencia. Asimismo, se utilizó la prueba Chi-cuadrado para comparar la frecuencia observada de la responsabilidad ante el reconocimiento social de graduación por los estudiantes considerándose el nivel de confianza al $95 \%$.

Aspectos éticos

Se consideró como aspectos éticos de la investigación lo siguiente:

\section{Derechos}

a) Consideración del carácter individual a participar en el estudio una vez que los objetivos y los métodos hayan sido explicados.

b) Necesidad social para comunicar los resultados contribuyendo a la visibilidad institucional.

c) Sugerencia de negación, a participar después de señaladas las condiciones para realizar el estudio.

2. Deberes

a) Informar a los participantes, la publicación del artículo científico.

b) Excluir toda posibilidad de engaños indebidos, influencia o intimidación.

\section{RESULTADOS}

Se propuso para la capacitación con modalidad acelerada lo siguiente:
Título:

- Proyecto de Tesina y Tesis de Investigación Científica para Estudiantes Universitarios

Fundamentación

- La metodología de la investigación científica, representa una herramienta de confiabilidad para la obtención y aplicación del conocimiento científico, la cual se basa en procesos estructurados y secuenciales que permiten construir la información sobre la base del razonamiento lógico como tipo de aprendizaje superior, a la hora de visualizar la relación sujeto - objeto - sujeto de investigación, además, del análisis sobre la propia complejidad de los fenómenos o sucesos que caracterizan al objeto de investigación.

- Para alcanzar la credibilidad científica de los resultados, es requerido el reconocimiento de transición sobre la información, siendo posible en el caso de los estudiantes universitarios a partir, del perfil de proyecto; y luego, el proyecto tesis de investigación.

\section{$\underline{\text { Objetivo general }}$}

- Evaluar las estructuras metodológ0icas de los formatos de tesina y tesis de investigación científica.

\section{$\underline{\text { Objetivos específicos }}$}

1. Gestión y administración de bases de datos científicas: Scielo, Scopus.

2. Seleccionar la publicación científica según bases de datos prestigio internacional.

3. Reconocer el silogismo deductivo e inductivo que caracterizan a las investigaciones con enfoque cuantitativo y cualitativo.

$\underline{\text { Temario }}$

1. Acceso al gestor Mendeley. Búsqueda de información científica y descarga de artículos científicos: bases de datos Scielo y Sciencedirect.

2. Administración de referencias bibliográficas. Organización de ficheros. Generación de bibliográficas e inserción de citas bibliográficas hacia el manuscrito. 
3. Estilos y conversión de citas bibliográficas: formato APA, edición $6^{\text {ta }}$.

4. Enseñanza secuencial del proceso de investigación: protocolo de investigación, perfil de tesis de investigación y proyectos de tesis de investigación.

5. Silogismo de las investigaciones cuantitativas y cualitativas. Situación problemática y problema científico: tipos y estructura. Preguntas y justificación de la investigación.

6. Formulación y clasificación de hipótesis, variables, métodos de estudios cuantitativos y cualitativos.
7. Tipos de muestreos probabilísticos y no probabilísticos. Técnicas de investigaciones cuantitativas y cualitativas.

8. Normalidad de los datos. Pruebas paramétricas: pruebas $t$-Student, análisis de la varianza (simple y multifactorial).

9. Análisis de regresión simple y múltiple.

Se muestra la responsabilidad ante el reconocimiento social de graduación donde la asistencia y el avance sobre las actividades prácticas desarrolladas en clase por los estudiantes disminuyeron por día en la réplica I pero se mantuvieron en la réplica II (Tabla 2).

Tabla 2. Responsabilidad ante el reconocimiento social de graduación $=\mathrm{RRSG} /$ Asistencia $=\mathrm{A}$. AAPPD $=$ avance sobre actividades prácticas desarrolladas en clase.

\begin{tabular}{ccccccc}
\hline Réplica & RRSG & Lunes & Martes & Miércoles & Jueves & Viernes \\
\hline I & A & 50 & 26 & 18 & 12 & 4 \\
& AAPDC & 100 & 90 & 30 & 14 & 3 \\
II & A & 18 & 18 & 18 & 18 & 18 \\
& AAPDC & 100 & 100 & 100 & 100 & 100 \\
\hline
\end{tabular}

Se muestra el número de propuestas de proyectos a presentar (último día: viernes) donde se obtuvo, un criterio de evaluación baja para la réplica I y alta en la réplica II (Tabla 3).

Tabla 3. Número de propuestas de proyectos a presentar $=$ NPPP $/$ Porcentaje $=\% /$ Criterio de evaluación $=$ CE.

\begin{tabular}{ccccc}
\hline Réplica & Día & NPPP & $\%$ & CE \\
\hline I & Viernes & 3 & 6 & baja \\
II & & 18 & 100 & alta
\end{tabular}

Se muestra la frecuencia observada y el porcentaje por réplicas del curso. La prueba de independencia indicó que, el valor de $\mathrm{p}$ fue menor que 0,05 por cuanto, no se puede rechazar la hipótesis que, el número de propuesta de proyectos a presentar (NPPP) depende de la responsabilidad ante el reconocimiento social de graduación (RRSG) por los estudiantes (nivel de confianza del 95\%) (Tabla 4).

Tabla 4. Frecuencia / Prueba de independencia.

\begin{tabular}{|c|c|c|c|c|c|c|c|}
\hline & Réplic: & & $\%$ & Réplica II & $\%$ & $\begin{array}{c}\text { Total por } \\
\text { Fila }\end{array}$ & $\%$ \\
\hline alta & 50 & & 65,79 & 18 & 23,68 & 68 & 89,47 \\
\hline baja & 3 & & 3,95 & 5 & 6,58 & 8 & 10,53 \\
\hline Total por columna & 53 & & 69,74 & 23 & 30,26 & 76 & 100,00 \\
\hline Prueba & Estadístico & Gl & Valor P & & & & \\
\hline Chi-Cuadrada & 4,403 & 1 & 0,0359 & & & & \\
\hline
\end{tabular}


Ante los resultados de la réplica I se reconsideró, cómo empezar la modalidad acelerada donde cuatro rutas de planificación fueron planteadas siendo la ruta número D (producción e investigación) quien posibilitó el desarrollo formativo del aprendizaje (Fig. 1).

Ruta A: Reconocimiento de las líneas de investigación / Facultad de Ingeniería Civil

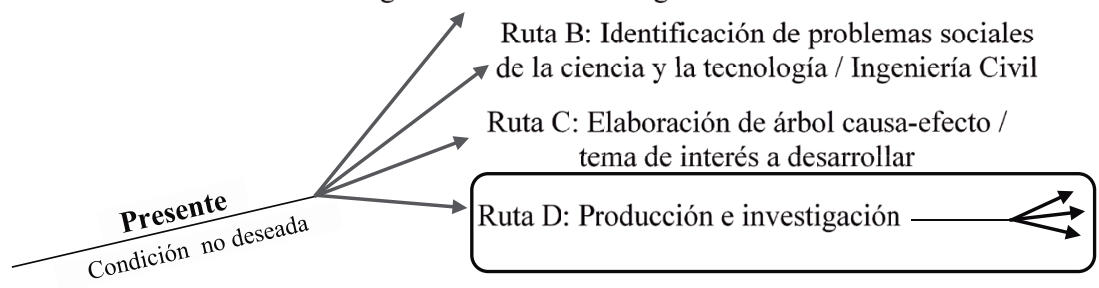

Figura 1. Rutas de la planificación prospectiva para el desarrollo formativo del aprendizaje.

La figura 2 muestra, las interrogantes para la producción e investigación con relación a la metodología de la investigación científica bajo la modalidad acelerada donde la tenencia de instrumentos de medición (¿qué se tiene?) garantiza la selección de las variables como tema de investigación pretendido a desarrollar.

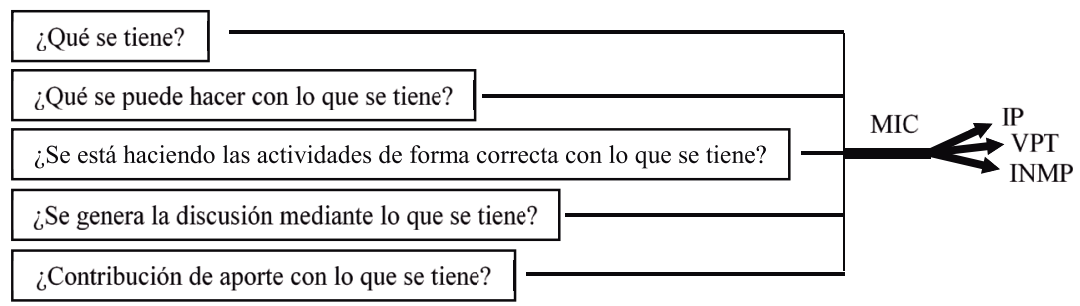

Figura 2. Interrogantes para la producción e investigación / metodología de la investigación científica = MIC / Implementación de procesos = IP / Validación de procesos técnicos = VPT / Introducción de nuevos y mejorados productos = INMP.

\section{DISCUSIÓN}

El escenario de la responsabilidad social, es un tema destacable en las universidades (Días \& Facal, 2011). Aunque, ya se señala como funciones sustantivas de la universidad a la docencia, investigación, extensión y gestión (Vallaeys, 2008) de la misma manera, debe incluirse una quinta que estaría relacionada con la orientación preparatoria selectiva para la exportación social de egresados competentes. En tal sentido, se observó al finalizar la réplica I que, la asistencia y el avance sobre actividades prácticas desarrolladas en clase fue disminuyendo lo cual denotó, irresponsabilidad de los estudiantes universitarios para que comprendieran el significado de alcanzar su título profesional al culminar de forma inmediata el último ciclo académico. Sin embargo, tal comportamiento fue diferente para la réplica II donde se reconoció a partir, de la exploración sobre cuatro rutas de planificación prospectiva para el desarrollo formativo del aprendizaje que, la producción e investigación resultó la más satisfactoria y donde los estudiantes universitarios pudieron comprender mediante cinco interrogantes desde lo que se tiene (instrumento de medición para sus variables) hasta su diferenciación temática (según los criterios o valores potenciales de investigación científica).

Una de las principales dificultades observadas en los estudiantes universitarios fue, la limitada preparación metodológica orientada a la investigación científica y donde probablemente, existió un vacío en la responsabilidad formativa de los docentes (fue mencionado por los estudiantes aunque no se midió) y tal como señala Peralta (2016), el profesor resulta el factor clave para lograr la calidad del aprendizaje.

En la literatura científica, existen diversos registros que abordan la estructura sobre metodología de la investigación científica y detallan en sí mismo su esencia (Blaxter et al., 2008; Cornejo et al., 2008; Tam et al., 2008; Hernández et al., 2010; Arévalo, 2013; 
Enríquez \& Argota, 2016). Sin embargo, al parecer resulta complejo entender, qué estrategia elaborar para que los estudiantes no solo comprendan el proceso de investigación sino, la relevancia científica de su tema de selección. Considerando lo planteado por Argota et al. (2019) que el aprendizaje de procesos metodológicos se adquiere mediante prácticas evaluativas se reformuló un modelo de pensamiento el cual, expresara por cada temática, evaluaciones relacionadas a la estructura del perfil de proyecto tesis (plan de tesis) y al mismo tiempo, garantizar que los estudiantes se familiaricen con lo pretendido a realizar pero reconociéndose la estructura metodológica acorde al proceso de lo que se identifica.

La principal limitación del estudio fue la puntualidad horaria de los estudiantes donde algunas prácticas colectivas para la evaluación y certificación del aprendizaje no se desarrollaron (Ramirez \& Zwerg, 2012).

Se concluyó que, la planificación acelerada de cuatro horas y durante cinco días a la semana comenzando por reconocer las variables y su posibilidad inmediata de medición garantizó, el desarrollo formativo del aprendizaje sobre la metodología de la investigación científica en estudiantes universitarios.

\section{AGRADECIMIENTOS}

A los estudiantes del $10^{\mathrm{mo}}$ ciclo académico de la carrera profesional: Ingeniería Civil de la Universidad Nacional "San Luis Gonzaga" por su reconocimiento de necesidad hacia una capacitación garantizada y compromiso a la divulgación de los resultados que sean contribuyente a dimensionar la visibilidad institucional.

\section{REFERENCIAS BIBLIOGRÁFICAS}

Abrahamson, E. 1991. Managerial fads and fashions: the diffusion and rejection of innovations. Academy of Management Review, 16: 586-612.

Anderson, R.J. 2012. Imagining novel futures: the roles of event plausibility and familiarity. Memory, 20: 443-451.

Arévalo, B.A.R. 2013. Cómo hacer un trabajo de investigación. Revista Médica La Paz, 19: 72-78.

Argota, P.G.; Gorina, S.A.; Solano, G.C.; Córdova, S.C.; Yallico, C.R.M. \& Pérez, C.B. 2019. Aprendizaje de procesos metodológicos mediante prácticas evaluativas en docentes universitarios. Revista The Biologist (Lima), 17: 125-133.

Mera, R.C. 2014. Pensamiento prospectivo: visión sistémica de la construcción del futuro. Revista Colombiana de Humanidades Análisis, 46: 89114.

Mattar, J. \& Cuervo, M. 2017. Planificación y prospectiva para la construcción de futuro en América Latina $y$ el Caribe. Textos seleccionados. CEPAL. Naciones Unidas. Santiago.

Blaxter, L.; Hughes, C. \& Tight, M. 2008. Cómo se investiga: Crítica y fundamentos, pp. 37-38. Ed. GRAÓ, de IRIF, S.L. Barcelona, España.

Bradfield, R.; Derbyshire, J. \& Wright, G. 2016. The critical role of history in scenario thinking: augmenting causal analysis within the intuitive logics scenario development methodology. Futures, 77: 56-66.

Carless, D. 2011. From testing to productive student learning: Implementing formative assessment in Confucian-heritage settings. New York, NY: Routledge.

Cornejo, M.; Mendoza, F. \& Rojas, R.C. 2008. La investigación con relatos de vida: pistas y opciones del diseño metodológico. Revista Psykhe, 17: 29-39.

Cummings, S. \& Daellenbach, U. 2009. A guide to the future of strategy? The history of long range planning. Long Range Plan, 42: 234-263.

De Jouvenel, B. 1964. L'art de la Conjecture. Éd. du Rocher, Monaco/Sédeis (Société d'étude et de documentation économiques, industrielles et sociales), Futuribles, Paris.

Díaz, M.M. \& Facal, S.S. 2011. Percepciones de los estudiantes de la Facultad de Ciencias Empresariales sobre la responsabilidad social universitaria (Montevideo-Uruguay). Investigación \& Desarrollo, 19: 340-365.

Enríquez, S.P. \& Argota, P.G. 2016. Descripción interpretativa para la elaboración del perfil de tesis de investigación científica con enfoque cualimétrico (mixto). Revista Campus, 22: 151-164. 
Fisher, A.G. 2018. Planning Your Way: How Humans Strategically Evaluate Prospective Decisions. Neuron, 99: 874-876.

Gill, J. \& Whittle, S. 1993. Management by panacea: accounting for transience. Journal of Management Studies, 30: 281-295.

Godet, M. \& Durance, P. 2011. La prospectiva estratégica para las empresas y los territorios. Cap 2: De los problemas a los métodos, pp. 53-84. Organización de las Naciones Unidas para la Educación, la ciencia y la cultura.

Hansen, G. \& Ringdal, R. 2018. Formative assessment as a future step in maintaining the mastery-approach and performance-avoidance goal stability. Studies in Educational Evaluation, 56: 59-70.

Hernández, R.; Fernández, C. \& Baptista, P. 2010. Metodología de la investigación. Capítulo I, pp 2-16. El proceso de investigación y los enfoques cuantitativos y cualitativos hacia un modelo integral. $5^{\text {ta }}$ Ed. McGraw-Hill Interamericana.

Hoffmann, L.M.A. \& Koifman, L. 2013. O olhar supervisivo na perspectiva da ativação de processos de mudança. Physis, 23: 573-587.

Ingvar, D.H. 1985. Memory of the future: an essay on the temporal organization of conscious awareness. Human Neurobiology, 4: 127-136.

Jiménez, C.W. 1982. Introducción al Estudio de la Teoría Administrativa. México: FCE.

Lisle, J. 2015. Installing a system of performance standards for National Assessments in the Republic of Trinidad and Tobago: issues and challenges. Applied Measurement in Education, 28: 308-329.

Luo, L. 2018. Experiencing evidence-based library and information practice (EBLIP): Academic Librarians' perspective. College \& Research Libraries, 79: 554-567.

MacKay, D. \& McKiernan, P. 2016. Strategic Sense Giving from the Periphery: What Top Management Does Not Hear. Working Paper, University of Strathclyde.

Martin, B. 1995. Foresight in Science and Technology en Technology. Analysis \& Strategic Management, 7: 139-178.
Matus, C. 1972. Estrategia y Plan. Textos del ILPES Editorial Universitaria Siglo XXI Ed. Santiago de Chile.

McLelland, V.C.; Devitt, A.L.; Schacter, D.L. \& Addis, D.R. 2015. Making the future memorable: the phenomenology of remembered future events. Memory, 23: 1255-1263.

Mojica, F. 2006. Concepto y aplicación de la prospectiva estratégica. Revista Médica, 14: 122-131.

Patel, A. 2016. Gaining insight: re-thinking at the edge. Technological Forecasting and Social Change, 107: 141-153.

Peralta, C. 2016. Adecuación de la planeación didáctica como herramienta docente en un modelo universitario orientado al aprendizaje. Revista Electrónica Iberoamericana sobre Calidad, Eficacia y Cambio en Educación, 14: 109-130.

Ramírez, A.F.H. \& Zwerg, V.A.M. 2012. Metodología de la investigación: más que una receta. ADminister, 91-111.

Tam, J.; Vera, G. \& Olivero, R.R. 2008. Tipos, métodos y estrategias de investigación. Revista Pensamiento y Acción, 145-154.

Travis, E.; Woodhouse, S.; Tan, R.; Patel, S.; Donovan, J. \& Brogan, K. 2014. Operating theatre time, where does it all go? A prospective observational study. British Medical Journal, 349: 44-46.

Vallaeys, F. 2008. Responsabilidad social universitaria: Una nueva filosofía de gestión ética e inteligente para las universidades. Educación Superior y Sociedad, 12: 193-219.

Wang, J. \& Yang, K. 2014. Using type IV Pearson distribution to calculate the probabilities of underrun and overrun of lists of multiple cases. European Journal of Anaesthesiology, 31: 363370.

Yin, X. \& Buck, G.A. 2015. There is another choice: An exploration of integrating formative assessment in a Chinese high school chemistry classroom through collaborative action research. Cultural Studies of Science Education, 10: 719-752.

Received May 27, 2019.

Accepted October 26, 2019. 